\title{
Wideband Compact PI Equivalent Circuit for Modeling On-Chip Spiral Inductors
}

\author{
Yu-Shun Tsai and Tzyy-Sheng Horng, Senior Member, IEEE
}

\begin{abstract}
A novel wideband nine-element PI equivalent circuit is proposed for modeling on-chip deep-submicron spiral inductors. Mathematical formulations derived by analyzing three quality factors enable fast and accurate extraction of all model elements as frequency-independent values. The model was confirmed using three spiral inductors that were realized in a $90 \mathrm{~nm}$ CMOS process and compared with other models to demonstrate its superior accuracy.
\end{abstract}

Index Terms-Equivalent circuit model, quality factor, SPICEcompatible, spiral inductor.

\section{INTRODUCTION}

$\mathbf{M}$ ANY complex and difficult circuit analysis and design tasks are performed with CAD tools, which require accurate component models to achieve accurate circuit simulation results. Currently, the most common nine-element PI model of inductors [1] is narrow-banded. Available bandwidth in the model can be increased by using relatively more complex model configurations such as a modified T configuration [2], [3] or a two-PI configuration [4]. A simpler approach is increasing the number of elements in the PI model [4]-[7]. However, as elements are increased, model parameter extraction becomes more difficult and complex, potentially requiring time-consuming optimization. Modeling methods based on electromagnetic (EM) simulation have a large computational load [5]. Moreover, the resultant frequency-dependent physical models are difficult to integrate into SPICE-compatible simulators.

The elements in the wideband compact model proposed in this study (Fig. 1(a)) are identical to those in the PI model [1] except for an $R_{p}$ element representing high-frequency loss and an $L_{g}-C_{g}-R_{g}$ resonator representing higher-order transmission line effects on substrate impedance. The two-step model extraction procedure exploits three $Q$ factors to develop extraction formulations of model elements without optimization. The model can be integrated into SPICE-compatible simulators such that the values of its elements are independent of frequency. The proposed model was validated using CMOS spiral inductors with an octagonal layout, as shown in Fig. 1(b).

Manuscript received September 29, 2011; accepted November 16, 2011. Date of publication December 16, 2011; date of current version January 11, 2012. This work was supported by the National Science Council, Taiwan, under Grants 98-2218-E-230-001 and 99-2622-E-110-003-CC1, and by the Department of Industrial Technology, Taiwan, under Grant 99-EC-17-A-01-S1-104.

Y.-S. Tsai is with the Department of Electronic Engineering, Cheng Shiu University, Kaohsiung, Taiwan (e-mail: ystsai@csu.edu.tw).

T.-S. Horng is with the Department of Electrical Engineering, National Sun Yat-Sen University, Kaohsiung, Taiwan (e-mail: jason@ee.nsysu.edu.tw).

Digital Object Identifier 10.1109/LMWC.2011.2177650

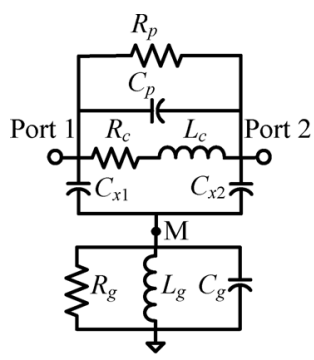

(a)

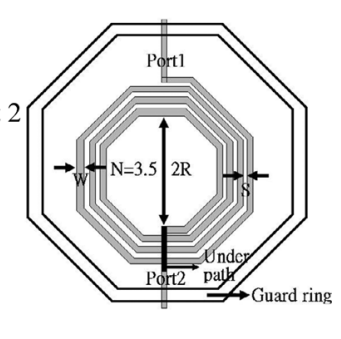

(b)
Fig. 1. Equivalent model for a $90 \mathrm{~nm}$ CMOS spiral inductor. (a) Proposed wideband equivalent circuit. (b) Layout of an octagonal shaped spiral inductor.

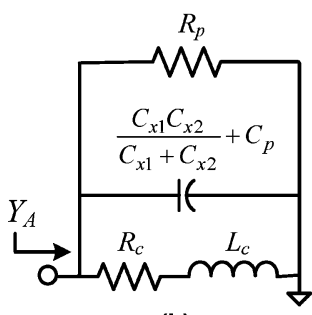

(b)

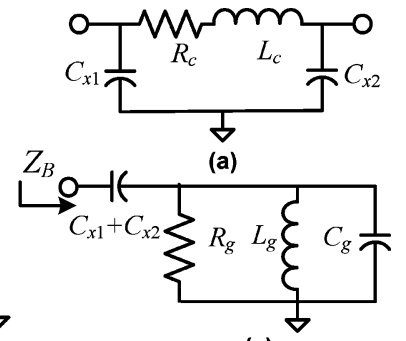

(c)
Fig. 2. Simplified and decomposed circuits. (a) The PI-equivalent circuit at low frequency. (b) The decomposed equivalent circuit with input admittance of $Y_{A}$ . (c) The decomposed equivalent circuit with input impedance of $Z_{B}$.

\section{Model Extraction Procedure}

\section{A. Step 1 Extraction at Low Frequency Condition}

When operating at a frequency much lower than either of the two resonant frequencies of $L_{c}, C_{p}$ and $L_{g},\left(C_{x 1}+C_{x 2}\right)$, the proposed model can be simplified as a simple PI model, as shown in Fig. 2(a). After the two-port measured $S$ parameters are converted into $Z$ and $Y$ parameters, the four elements in Fig. 2(a) can be determined from the following admittance parameters:

$$
\begin{aligned}
C_{x 1} & =\omega^{-1} \operatorname{Im}\left\{Y_{11}+Y_{12}\right\}, \\
C_{x 2} & =\omega^{-1} \operatorname{Im}\left\{Y_{22}+Y_{12}\right\}, \text { at low frequency } \\
Z_{c} & =R_{c}+j \omega L_{c}=-\frac{1}{Y_{12}},
\end{aligned}
$$

\section{B. Step 2 Extraction at High Frequency Condition}

The proposed model uses elements other than the above four elements to capture high frequency effects [6]. To simplify the extraction of these elements, the proposed model is decomposed 


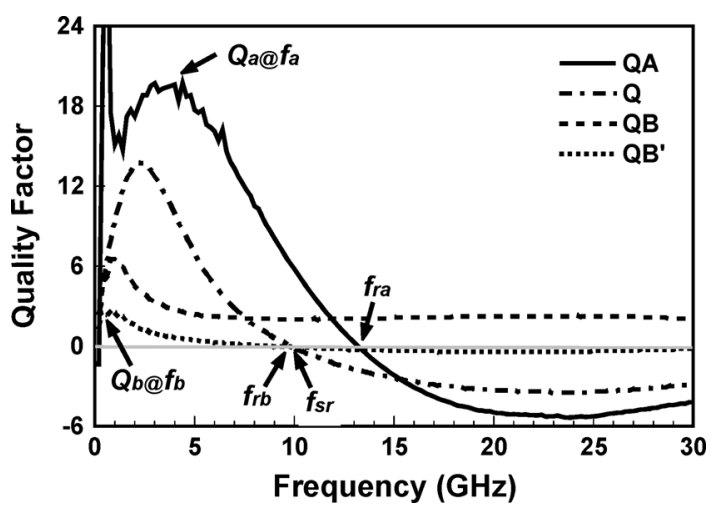

Fig. 3. Frequency responses of $Q, Q_{A}, Q_{B}$ and $Q_{B}^{\prime}$ factors for the $5 \mathrm{nH}$ CMOS spiral inductor.

according to the following relations into two one-port equivalent circuits [2], [3]:

$$
\begin{aligned}
Y_{A} & =Z_{A}^{-1}=\left(Z_{11}+Z_{22}-2 Z_{12}\right)^{-1} \\
& =\frac{1}{R_{c}+j \omega L_{c}}+j \omega C_{p}+\frac{1}{R_{p}}+j \omega\left(\frac{C_{x 1} C_{x 2}}{C_{x 1}+C_{x 2}}\right) \\
Z_{B} & =Y_{B}^{-1}=\left(Y_{11}+Y_{22}+2 Y_{12}\right)^{-1} \\
& =\left(j \omega C_{g}+\frac{1}{j \omega L_{g}}+\frac{1}{R_{g}}\right)^{-1}+\frac{1}{j \omega\left(C_{x 1}+C_{x 2}\right)} .
\end{aligned}
$$

Fig. 2(b) and (c) present the two one-port equivalent circuits with an input admittance of $Y_{A}$ and an input impedance of $Z_{B}$, respectively. By relating Fig. 1(a) to Fig. 2(b) and (c), $Y_{A}$ can be considered the input admittance as seen when looking into port 1 with an open circuit in node $M$ and a short circuit from port 2 to the ground, and $Z_{B}$ can be considered the input impedance as seen when looking into port 1 with a short circuit from port 2 to port 1 . With knowledge of the four elements $L_{c}, R_{c}, C_{x 1}$ and $C_{x 2}$, which were extracted from Step 1, the remaining five elements, $C_{p}, R_{p}, L_{g}, R_{g}$, and $C_{g}$, can be obtained using $Y_{A}$ and $Z_{B}$ from Step 2.

Another critical extraction procedure involves three $Q$ factors of an inductor, which are defined as

$$
\begin{aligned}
Q(\omega) & =-\frac{\operatorname{Im}\left\{Y_{11}(\omega)\right\}}{\operatorname{Re}\left\{Y_{11}(\omega)\right\}} \\
Q_{A}(\omega) & =-\frac{\operatorname{Im}\left\{Y_{A}(\omega)\right\}}{\operatorname{Re}\left\{Y_{A}(\omega)\right\}} \\
Q_{B}(\omega) & =-\frac{\operatorname{Im}\left\{Y_{B}(\omega)\right\}}{\operatorname{Re}\left\{Y_{B}(\omega)\right\}} .
\end{aligned}
$$

Fig. 3 presents the frequency responses of the three $Q$ factors for a $5 \mathrm{nH}$ CMOS spiral inductor. The self-resonant frequencies $\left(f_{r a}, f_{r b}\right.$, and $\left.f_{s r}\right)$ are determined from the zero positions of the $Q$ factors to extract the reactive elements. At the angular frequency $\omega_{r a}=2 \pi f_{r a}$, the imaginary part of (1) is zero, and solving this condition for $C_{p}$ yields

$$
C_{p}=\frac{1}{\omega_{r a}^{2} L_{c}}-\left(\frac{C_{x 1} C_{x 2}}{C_{x 1}+C_{x 2}}\right) .
$$

TABLE I

GeOMetrical Parameters AND THE EXTRACTED MOdEl PARAMETERS FOR THE THREE 90 NM CMOS SPIRAL INDUCTORS

\begin{tabular}{lccc}
\hline \hline $\begin{array}{l}\text { Geometrical and } \\
\text { model parameters }\end{array}$ & $\begin{array}{c}5-\mathrm{nH} \\
\text { inductor }\end{array}$ & $\begin{array}{c}3.3-\mathrm{nH} \\
\text { inductor }\end{array}$ & $\begin{array}{c}1.5-\mathrm{nH} \\
\text { inductor }\end{array}$ \\
\hline \hline $\mathrm{W}(\mu \mathrm{m})$ & 6 & 9 & 3 \\
$\mathrm{~S}(\mu \mathrm{m})$ & 3 & 3 & 3 \\
Number of turns & 3.5 & 3.5 & 3.5 \\
Inner diameter $(\mu \mathrm{m})$ & 90 & 60 & 15 \\
\hline \hline$L_{c}(\mathrm{nH})$ & 4.98 & 3.20 & 1.51 \\
$R_{c}(\Omega)$ & 3.05 & 1.84 & 2.87 \\
$C_{p}(\mathrm{fF})$ & 3.67 & 3.55 & 0.97 \\
$R_{p}(\Omega)$ & 2736 & 2172 & 2316 \\
$C_{x 1}(\mathrm{fF})$ & 52.5 & 48.2 & 15.2 \\
$C_{x 2}(\mathrm{fF})$ & 58.9 & 56.5 & 25.1 \\
$L_{g}(\mathrm{nH})$ & 2.19 & 2.51 & Not needed \\
$C_{g}(\mathrm{fF})$ & 107 & 116 & Not needed \\
$R_{g}(\Omega)$ & 55.1 & 52.6 & Not needed \\
\hline \hline
\end{tabular}

The admittance parameter $Y_{11}$ of the two-port inductor model neglecting the loss is

$$
\begin{aligned}
Y_{11}=\frac{1}{j \omega L_{c}}+j \omega\left(C_{p}+\right. & \left.\frac{C_{x 1} C_{x 2}}{C_{x 1}+C_{x 2}}\right) \\
& +\frac{\frac{\omega C_{x 1}{ }^{2} Y_{B}{ }^{\prime}}{C_{x 1}+C_{x 2}}}{\left(\omega\left(C_{x 1}+C_{x 2}\right)-j Y_{B}{ }^{\prime}\right)}
\end{aligned}
$$

where

$$
Y_{B}^{\prime}=j \omega C_{g}+\frac{1}{j \omega L_{g}} .
$$

The imaginary part of (10) vanishes at the angular frequency $\omega_{s r}=2 \pi f_{s r}$, and so

$$
\begin{aligned}
& L_{g} \\
& =\frac{\left(\omega_{s r}^{2}-\omega_{r b}^{2}\right)\left(\omega_{s r}^{2} L_{c}\left(C_{x 1}+C_{p}\right)-1\right)}{\omega_{s r}^{2} \omega_{r b}^{2}\left(C_{x 1}+C_{x 2}-\omega_{s r}^{2} L_{c}\left(C_{x 1} C_{x 2}+C_{p}\left(C_{x 1}+C_{x 2}\right)\right)\right)} .
\end{aligned}
$$

Since $\omega_{r b}=2 \pi f_{r b}$ is the angular resonant frequency of the $L_{g}-R_{g}-C_{g}$ resonator, $C_{g}$ can be further determined using

$$
C_{g}=\frac{1}{\omega_{r b}^{2} L_{g}} .
$$

With respect to the resistive elements, the peak $Q_{A}$ factor value $Q_{a}$ at the angular frequency $\omega_{a}=2 \pi f_{a}$ is used to extract $R_{p}$ as follows:

$$
R_{p}=\frac{Q_{a}\left(R_{c}^{2}+\omega_{a}^{2} L_{c}^{2}\right)}{Q_{a} R_{c}+\omega_{a} L_{c}-\omega_{a}\left(R_{c}^{2}+\omega_{a}^{2} L_{c}^{2}\right)\left(C_{p}+\frac{C_{x 1} C_{x 2}}{C_{x 1}+C_{x 2}}\right)} .
$$

In Fig. 3, $Q_{B}^{\prime}$ is defined as the quality factor of the $L_{g}-C_{g}-R_{g}$ resonator. Similarly, the peak $Q_{B}^{\prime}$ factor value $Q_{b}$ at the angular frequency $\omega_{b}=2 \pi f_{b}$ is used to extract $R_{g}$ as follows:

$$
R_{g}=\frac{Q_{b} \omega_{b} L_{g}}{1-\omega_{b}^{2} C_{g} L_{g}} .
$$




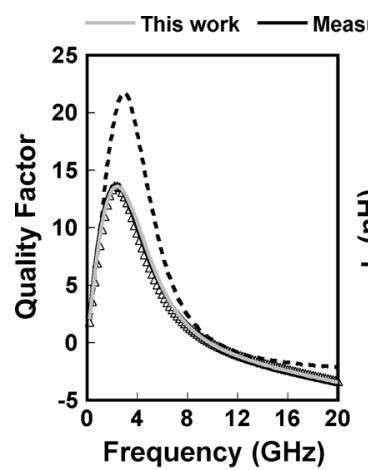

(a)

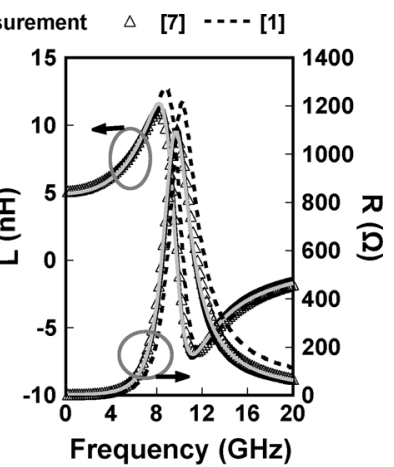

(b)
Fig. 4. Comparison of (a) quality factor and (b) series inductance and resistance, between measurement and the proposed model against the models studied in [1] and [7] for the $5 \mathrm{nH} 90 \mathrm{~nm}$ CMOS spiral inductor.

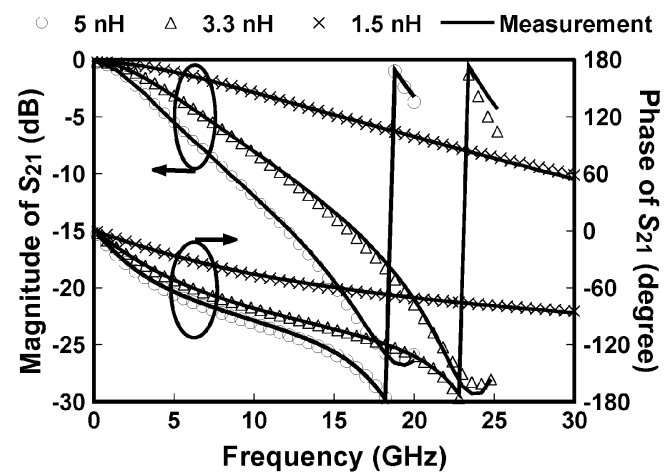

(a)

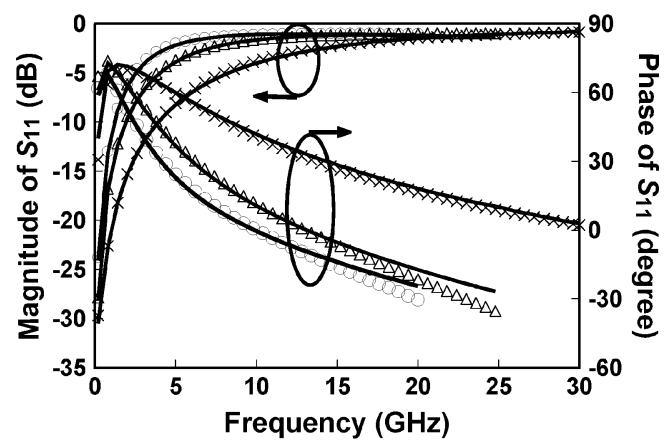

(b)

Fig. 5. Measured and modeled results for the magnitude of $S$-parameters, where black lines are measured results and symbols are modeled results. (a) $S_{21}$ (b) $S_{11}$

\section{MODEL VERIFICATION AND DISCUSSION}

Table I lists the layout parameters and the model parameters extracted using the proposed method for the three spiral inductors realized in a $90 \mathrm{~nm}$ single-poly nine-metal (1P9M) CMOS process. The substrate is $300 \mu \mathrm{m}$ thick silicon with a high resistivity of $0.1 \Omega \mathrm{m}$. Above the substrate is $0.35 \mu \mathrm{m}$ thick field oxide. The metal layers are copper. The inductor uses the top metal layer with a thickness of $3.3 \mu \mathrm{m}$ for winding and the second top metal layer with a thickness of $0.8 \mu \mathrm{m}$ for an underpath. The inter-metal dielectric (IMD) is $10 \mu \mathrm{m}$ thick with a dielectric constant of 4.2. Notably, the high-resistivity silicon substrate makes the internal inductance associated with the silicon substrate nearly invariant with frequency. Therefore, the extracted inductances in the model can be considered frequency independent over a wide frequency range [8].

To compare the proposed model with other reported models and measurement, Fig. 4(a) and (b) present the modeled $Q$ factor and the extracted inductance and resistance, respectively, of the $5 \mathrm{nH}$ CMOS inductor. Clearly, the nine-element PI model described by Lee [1] is less accurate compared to the 12-element model presented by Chen [7]. The proposed nine- element model achieves comparable or even better performance mainly because the $L_{g}-C_{g}-R_{g}$ resonator functions as the additive term for a third-order approximation of the transmission-line effects on the substrate impedance. Notably, a similar method devised by Lee [6] established a wideband equivalent circuit for the intrinsic inductor impedance. Based on the values extracted for the elements presented in Table I, the modeled and measured $S$-parameters in Fig. 5 confirm the accuracy of the proposed model.

\section{CONCLUSION}

The proposed model uses only nine elements, which is the same number used in the conventional PI model, but it achieves relatively higher wideband accuracy. Good agreement between measured and modeled results validates the proposed model.

\section{REFERENCES}

[1] T. H. Lee, The Design of CMOS Radio-Frequency Integrated Circuits. New York: Cambridge Univ. Press, 1988.

[2] T.-S. Horng, J.-M. Wu, L.-Q. Yang, and S.-T. Fang, "A novel modified-T equivalent circuit for modeling LTCC embedded inductors with a large bandwidth," IEEE Trans. Microw. Theory Tech., vol. 51, no. 12, pp. 2327-2333, Dec. 2003.

[3] Y.-S. Tsai, H.-W. Chou, Y.-C. Lin, and T.-S. Horng, "Accurate modeling of RF passive component in deep submicron process," in Proc. Int. Conf. Appl. Electromag. Student Innovation Competition Awards, 2010, pp. 167-171.

[4] I. C. H. Lai and M. Fujishima, "A new on-chip substrate-coupled inductor model implemented with scalable expressions," IEEE J. SolidState Circuits, vol. 41, no. 11, pp. 2491-2499, Nov. 2006.

[5] J. Brinkhoff, K. S. S. Koh, K. Kang, and F. Lin, "Scalable transmission line and inductor models for CMOS millimeter-wave design," IEEE Trans. Microw. Theory Tech., vol. 56, no. 12, pp. 2954-2962, Dec. 2008.

[6] K.-Y. Lee, S. Mohammadi, P. K. Bhattacharya, and L. P. B. Katehi, "A wideband compact model for integrated inductors," IEEE Microw. Wireless Compon. Lett., vol. 16, no. 9, pp. 490-492, Sep. 2006.

[7] H.-H. Chen, H.-W. Zhang, S.-J. Chung, J.-T. Kuo, and T.-C. Wu, "Accurate systematic model-parameter extraction for on-chip spiral inductors," IEEE Trans. Microw. Theory Tech., vol. 55, no. 9, pp. 3267-3273, Sep. 2008.

[8] H. Ymeri, B. Nauwelaers, K. Maex, D. De Roest, M. Stucchi, and S. Vandenberghe, "Frequency-dependent internal inductance associated with silicon substrate of on-chip interconnects," in Proc. Int. Sci. Conf. (RADIOELEKTRONIKA), Bratislava, Slovak Republic, 2002, pp. 14-16. 